\title{
Quantitative interaction screen of telomeric repeat- containing RNA reveals novel TERRA regulators
}

\author{
Marion Scheibe, ${ }^{1,4}$ Nausica Arnoult, ${ }^{2,4}$ Dennis Kappei, ${ }^{3}$ Frank Buchholz, ${ }^{3}$ \\ Anabelle Decottignies, ${ }^{2}$ Falk Butter, ${ }^{1,5}$ and Matthias Mann ${ }^{1,5}$ \\ ${ }^{7}$ Department of Proteomics and Signal Transduction, Max Planck Institute of Biochemistry, 82152 Martinsried, Germany; ${ }^{2}$ de Duve \\ Institute, Catholic University of Leuven, 1200 Brussels, Belgium; ${ }^{3}$ Department of Medical Systems Biology, University Hospital \\ and Medical Faculty Carl Gustav Carus, TU Dresden, 01307 Dresden, Germany
}

\begin{abstract}
Telomeres are actively transcribed into telomeric repeat-containing RNA (TERRA), which has been implicated in the regulation of telomere length and heterochromatin formation. Here, we applied quantitative mass spectrometry (MS)-based proteomics to obtain a high-confidence interactome of TERRA. Using SILAC-labeled nuclear cell lysates in an RNA pulldown experiment and two different salt conditions, we distinguished 115 proteins binding specifically to TERRA out of a large set of background binders. While TERRA binders identified in two previous studies showed little overlap, using quantitative mass spectrometry we obtained many candidates reported in these two studies. To test whether novel candidates found here are involved in TERRA regulation, we performed an esiRNA-based interference analysis for 15 of them. Knockdown of 10 genes encoding candidate proteins significantly affected total cellular levels of TERRA, and RNAi of five candidates perturbed TERRA recruitment to telomeres. Notably, depletion of SRRT / ARS2, involved in miRNA processing, up-regulated both total and telomere-bound TERRA. Conversely, knockdown of MORF4L2, a component of the NuA4 histone acetyltransferase complex, reduced TERRA levels both globally and for telomere-bound TERRA. We thus identified new proteins involved in the homeostasis and telomeric abundance of TERRA, extending our knowledge of TERRA regulation.
\end{abstract}

[Supplemental material is available for this article.]

The ends of linear chromosomes are protected from fusions and degradation by specialized structures called telomeres, composed of repetitive DNA and bound by the six-protein complex shelterin (Palm and de Lange 2008). In mammals, telomeric DNA consists of double-stranded TTAGGG repeats ending in a single-stranded G-rich overhang. Telomeric regions are transcribed by RNA polymerase II, starting from subtelomeric CpG-island promoters and extending toward the telomeric tract. This active transcription produces G-rich telomeric repeat-containing RNA (TERRA) (Azzalin et al. 2007; Schoeftner and Blasco 2008). TERRA length is proportional to telomere length, and a fraction of the cellular TERRA pool is bound to telomeres (Nergadze et al. 2009; Porro et al. 2010; Arnoult et al. 2012). TERRA levels are cell cycle-regulated and their transcription is repressed by promoter methylation (Nergadze et al. 2009) and repressive histone marks at longer telomeres (Arnoult et al. 2012).

From a functional point of view, compelling evidence suggests that TERRA participates in telomeric heterochromatin formation by promoting H3K9 trimethylation (Deng et al. 2009; Arnoult et al. 2012). This noncoding RNA species may also be involved in telomere length regulation by counteracting telomere extension by telomerase (Redon et al. 2010), although these data obtained in vitro were not confirmed in human cancer cells (Farnung et al. 2012). In budding yeast, up-regulation of telomere transcription promotes telomere degradation (Pfeiffer and Lingner 2012). Some of the other proposed functions of TERRA include regulation of telomere motion (Arora et al. 2012) and telomere

\footnotetext{
${ }^{4}$ These authors contributed equally to this work.

${ }^{5}$ Corresponding authors

E-mail butter@biochem.mpg.de

E-mail mmann@biochem.mpg.de

Article published online before print. Article, supplemental material, and publication date are at http://www.genome.org/cgi/doi/10.1101/gr.151878.112.
}

capping by orchestrating an RPA-to-POT1 switch during cell cycle progression (Flynn et al. 2011). While clearly the functional understanding of TERRA is currently just at its beginning, TERRA seems to achieve its function through the recruitment of specific proteins to telomeres, such as ORCs (Deng et al. 2009) and HNRNPA1 (Flynn et al. 2011) in human or Exo1 in yeast (Pfeiffer and Lingner 2012). Comprehensive identification of TERRA binding proteins is therefore of major importance for a better understanding of the biological functions of this noncoding RNA.

To identify TERRA-bound proteins, previous studies relied on RNA pull-downs combined with mass spectrometric analysis of selected protein bands present in TERRA pull-downs compared with a control sequence or nonquantitative analysis. Deng et al. (2009) identified two ORC subunits, ORC1 and ORC5, and showed that the ORC complex is recruited to TERRA by TERF2. A separate mass spectrometric analysis mostly identified heterogeneous nuclear ribonucleoproteins (HNRNPs) as TERRA partners and focused on the characterization of HNRNP function in TERRA regulation in mouse fibroblasts (Lopez de Silanes et al. 2010). However, there was almost no overlap in the proteins identified by mass spectrometry in these two studies. While these studies were performed with extracts from Raji cells and primary mouse embryonic fibroblasts (MEFs), respectively, most of the identified proteins are ubiquitously expressed and thus should have been detected in both experiments. To address this discrepancy and to identify novel TERRA-associated proteins without relying on visual differences in Coomassie- or silver-stained gels, we performed RNA 
pull-down experiments combined with quantitative mass spectrometry using stable isotope labeling by amino acids in cell culture (SILAC) (Butter et al. 2009; Scheibe et al. 2012). This powerful technique allows us to quantify differences in the enrichment of hundreds of proteins on an RNA sequence of interest versus a control sequence. SILAC determines the intensity of specific "light" and "heavy" peptide pairs that are mass shifted from each other due to metabolic labeling of the proteome with isotopeenriched amino acids (Mann 2006). So far, SILAC-based quantitative mass spectrometry for the identification of RNA-associated proteins has been used to investigate selected mRNA fragments (Butter et al. 2009; Tsai et al. 2011; Ward et al. 2011; Scheibe et al. 2012) or polyadenylated RNA on a global scale (Baltz et al. 2012). For the first time, we here apply quantitative interaction proteomics to systematically and comprehensively determine proteins interacting with a noncoding repetitive RNA, leading to the identification of novel interactors with a role in TERRA homeostasis and its association with telomeres.

\section{Result}

\section{Quantitative mass spectrometry screen}

To identify TERRA-interacting proteins, we used SILAC-based quantitative mass spectrometry to measure the enrichment of hundreds of proteins simultaneously. A TERRA (UUAGGG) 8 RNA oligonucleotide was synthesized by solid-phase chemistry and tagged with a biotin group at the $3^{\prime}$ end to serve as bait. An RNA oligonucleotide composed of the (GUGUGA) 8 shuffled repeat sequence was used as control (Fig. 1A). These constructs are of identical length as previously used in the other two screens for TERRA interactors (Deng et al. 2009; Lopez de Silanes et al. 2010). The RNA oligonucleotides were incubated with heavy lysine- and arginine-labeled nuclear extracts (see Methods). We also performed a second independent TERRA pull-down in which we switched the labels and thus are able to visualize the bound proteins in a twodimensional interaction plot (Butter et al. 2009; Ong 2010). Specific interactors of the TERRA RNA bait show an inverse ratio when they are incubated with the light SILAC extract (label switch) and are thus grouped to the upper left quadrant. Proteins binding unspecifically to the beads or the RNA show a one-to-one SILAC ratio and cluster around the origin of the plot.
We performed the experiment under mild washing conditions of $250 \mathrm{mM}$ sodium chloride to preserve weak interactions and quantified 790 protein groups with at least two independent SILAC ratio counts each (Fig. 1B). Of these proteins, 134 were at least fourfold enriched with the (UUAGGG) 8 probe in both the forward and the label-switch pull-down. Because of the mild washing conditions, we observed some variation in the SILAC ratios of the quantified proteins (Pearson coefficient 0.57 after filtering for contaminants) between both pull-downs. We reasoned that more stringent wash conditions might result in a better reproducibility between the pull-downs and therefore performed a second experiment, in which we increased the stringency of the washing conditions to $1 \mathrm{M}$ sodium chloride to eliminate interactors with lower affinity (Fig. 1C). As expected, this experiment resulted in a higher reproducibility of SILAC ratios between the two pull-downs (Pearson coefficient 0.90 after filtering for contaminants). Additionally, it increased the average SILAC ratios of the interacting proteins. This effect is presumably due to the removal of proteins binding unspecifically to the bead matrix, thus resulting in higher enrichment ratios in an environment of overall reduced background. We quantified 1003 proteins with two independent ratio counts, of which 400 were at least fourfold enriched (Supplemental Table 1). The larger number of identifications after washing with 1 $M$ sodium chloride might be due to a reduced dynamic range in the sample that is beneficial for mass spectrometric measurements.

When we compare the proteins enriched at both salt concentrations with a minimum of two quantitation events in at least one of the experiments, we found that 115 of them were enriched under both conditions. This filtered group was used for further analysis. To investigate possible physical or functional interactions of the enriched proteins, we analyzed them in the STRING environment (Szklarczyk et al. 2011). Interestingly, 67 of these 115 proteins formed a single STRING network that readily visualized different functional clusters and physical complexes (Fig. 2). The major clusters belong to chromatin remodeling (SWI-SNF and HDAC-associated complex), DNA replication (RFC), RNA degradation (exosome), transcription (RNA polymerases I, II, and III), and protein translation ( $43 \mathrm{~S}$ ribosome).

\section{Comparison to previous screens for TERRA interactors}

We compared our results with the reported interactors from the previous two mass spectrometric screens (Deng et al. 2009;
A

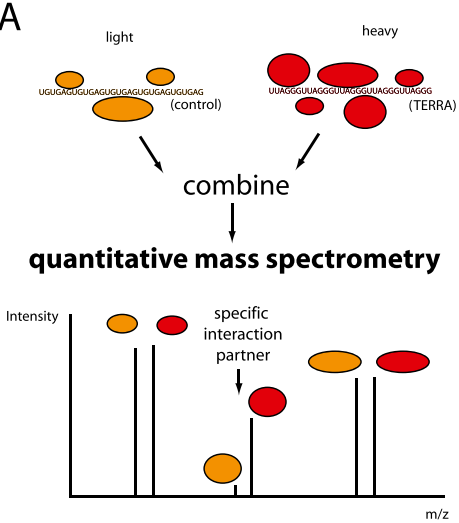

B

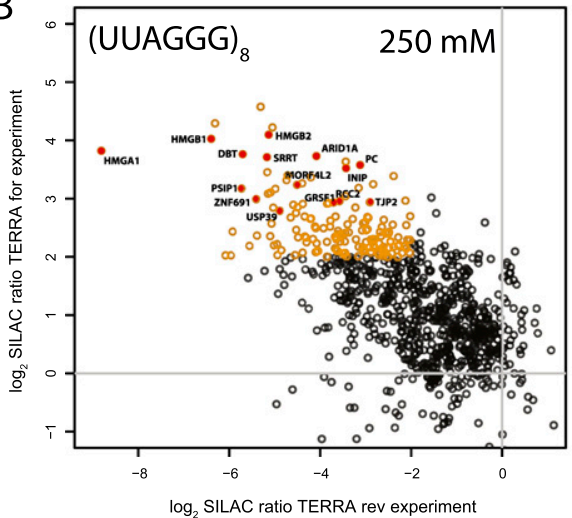

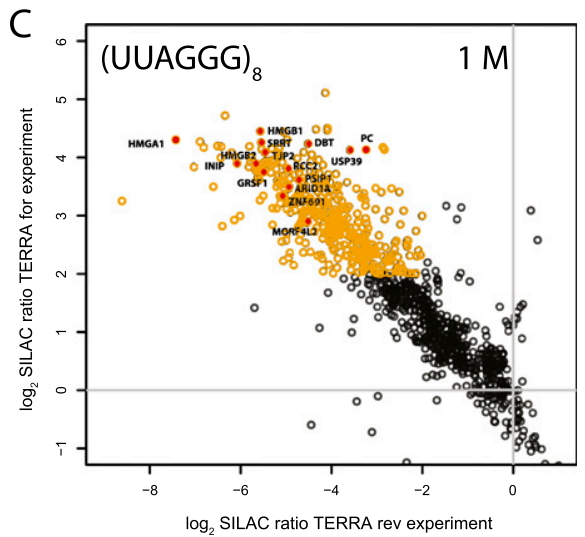

Figure 1. Schematic representation of the quantitative TERRA pull-down. (A) The nuclear extract of HeLa cells labeled by SILAC was incubated with immobilized UUAGGG-repeat-containing RNA or a shuffled control fragment. Both fractions were combined prior to quantitative mass spectrometry. $(B, C)$ Two-dimensional interaction plot for the TERRA pull-down under relatively mild ( $250 \mathrm{mM}$ sodium chloride) washing conditions $(B)$ and under more stringent (1 M sodium chloride) washing conditions (C). The 15 candidates (red-filled circles) selected for the secondary RNAi screen are annotated.

2150 Genome Research www.genome.org 


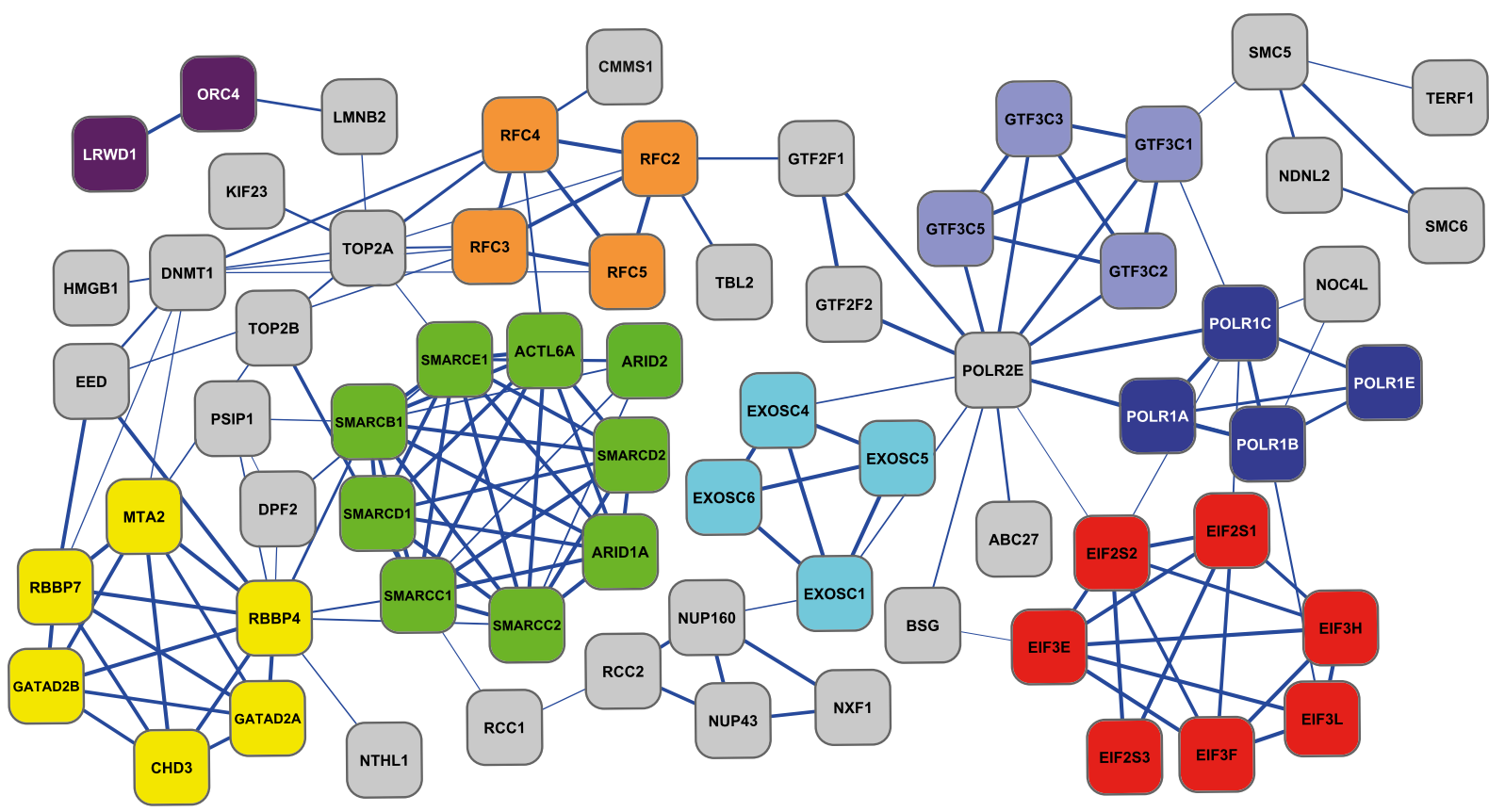

Figure 2. Proteins with a relative enrichment of at least fourfold both under mild and stringent washing conditions were investigated for functional protein interactions using the STRING database. Of the 115 significantly enriched proteins, 67 proteins form a functional network containing several established physical complexes: exosome (cyan), polymerase I (dark blue), TFIIIC (light blue), eukaryotic initiations factors (red), replication factor complex (orange), origin recognition complex (violet), SWI-SNF (green), and HDAC-associated complex (yellow).

Lopez de Silanes et al. 2010). In those experiments, the investigators relied on the selection of gel bands by visual inspection or nonquantitative measurements and reported 26 (Deng et al. 2009) and 41 proteins (Lopez de Silanes et al. 2010) to be enriched at $(\mathrm{UUAGGG})_{8}$ repeats compared with their control sequence. However, only HNRNPA1 and HNRNPM, two highly abundant nuclear proteins, were commonly identified in both studies. Because most of the proteins found in the two studies are ubiquitously expressed, this small overlap is unexpected. From the combined 65 proteins identified in the previous studies, we obtained SILAC ratios for 51 . At $250 \mathrm{mM}$ sodium chloride, we determined seven to be specific (enriched at least four times) for our (UUAGGG) ${ }_{8}$ oligonucleotide, while 16 were moderately enriched for (UUAGGG) ${ }_{8}$ in our setup with SILAC ratios between 1.5 and 4.0. These proteins may bind TERRA less specifically, considering that we found 134 proteins (at $250 \mathrm{mM}$ sodium chloride) with at least fourfold enrichment. While this number increases significantly to 21 specific and 37 moderately enriched when we consider the stringent wash (1 M sodium chloride), we also observed an overall increase in SILAC ratios for this condition. Thus, our data suggest that the HNRNPs and the ribosomal proteins that were reported previously to bind to TERRA may not be highly specific TERRA binders.

To estimate the comprehensiveness of our screen, we investigated how many subunits of complexes we were able to detect, based on the premise that all subunits of stable complexes should generally show coordinated behavior. In one of the previous screens, TJP1 was identified but TJP2 of the tight junction complex heterodimer was absent (Lopez de Silanes et al. 2010). Here, we identify both subunits with similar SILAC ratios $>4$. For the two ORC subunits, ORC1 and ORC5, found by mass spectrometry in one of the previous studies (Deng et al. 2009), we measured high SILAC ratios of 3.374.77 in the $250 \mathrm{mM}$ sodium chloride wash conditions and SILAC ratios of 3.41-8.53 in the $1 \mathrm{M}$ sodium chloride experiment. For ORC4, another subunit not identified in the previous mass spectrometric screen but shown to bind by immunostaining (Deng et al. 2009), we obtained a SILAC ratio $>4$ in both of our SILAC pull-down experiments. We also achieved good coverage of other physical protein complexes such as SWI-SNF (eight subunits), the exosome (four subunits), and the RFC complex (four subunits) in our set of specific binders. These results show that visual inspection of gel lanes is prone to underestimate enriched proteins, especially low abundant ones. We identify most of the proteins of the previous screens, especially when likely contaminants such as keratins are excluded, and cover most members of stable complexes that are enriched on TERRA. This suggests that our screen reaches considerably greater depth in identifying the interaction partners of TERRA than previously possible. It also increases confidence in the specificity of the novel proteins that we uncovered in our investigation for biological follow-up experiments.

\section{Selection of candidates from quantitative MS screen}

SILAC-based RNA-protein interaction analysis has already previously resulted in the identification of physiological relevant RNA-protein interactions (Butter et al. 2009; Ward et al. 2011; Scheibe et al. 2012; Klass et al. 2013). To start the functional assessment of specifically bound proteins, we selected candidates previously not associated with TERRA physiology among the topscoring proteins for an analysis by RNA interference (Fig. 1B,C; Table 1). We only chose proteins that were reproducibly highly enriched in the low-stringency wash ( $250 \mathrm{mM}$ sodium chloride) as well as in the high-stringency wash ( $1 \mathrm{M}$ sodium chloride). While necessary to reduce the candidates for the RNAi screen to a reasonable number, the lower-ranking proteins might still serve an important function in TERRA biology. Fifteen candidates fulfilling 
Table 1. Candidates consistently enriched in both pull-down experiments and investigated for their effect on TERRA levels and localization by an RNA interference screen

\begin{tabular}{|c|c|c|c|c|c|c|c|c|c|c|c|c|}
\hline \multirow[b]{2}{*}{ Protein } & \multicolumn{3}{|c|}{ Forward (250 mM NaCl) } & \multicolumn{3}{|c|}{ Reverse $(250 \mathrm{mM} \mathrm{NaCl})$} & \multicolumn{3}{|c|}{ Forward (1 M NaCl) } & \multicolumn{3}{|c|}{ Reverse ( $1 \mathrm{M} \mathrm{NaCl})$} \\
\hline & Peptides & Ratio & Quants & Peptides & Ratio & Quants & Peptides & Ratio & Quants & Peptides & Ratio & Quants \\
\hline ARID1A & 2 & 13.28 & 3 & 11 & 0.06 & 9 & 12 & 11.24 & 16 & 14 & 0.03 & 20 \\
\hline INIP & 3 & 11.50 & 2 & 1 & 0.09 & 2 & 3 & 14.91 & 4 & 5 & 0.01 & 6 \\
\hline DBT & 16 & 13.58 & 15 & 16 & 0.02 & 18 & 14 & 18.84 & 19 & 15 & 0.04 & 14 \\
\hline ZNF691 & 2 & 7.95 & 3 & 5 & 0.02 & 5 & 2 & 10.07 & 2 & 4 & 0.03 & 4 \\
\hline GRSF1 & 5 & 7.64 & 4 & 3 & 0.08 & 3 & 12 & 13.46 & 18 & 12 & 0.02 & 16 \\
\hline HMGA1 & 3 & 14.14 & 3 & 1 & 0.00 & 1 & 1 & 19.76 & 3 & 2 & 0.01 & 7 \\
\hline HMGB1 & 6 & 16.30 & 14 & 2 & 0.01 & 8 & 7 & 21.85 & 18 & 6 & 0.02 & 22 \\
\hline HMGB2 & 5 & 17.12 & 7 & 6 & 0.03 & 10 & 6 & 14.85 & 13 & 7 & 0.02 & 14 \\
\hline MORF4L2 & 3 & 9.42 & 3 & 1 & 0.04 & 1 & 2 & 7.45 & 3 & 2 & 0.04 & 2 \\
\hline PC & 8 & 11.94 & 8 & 3 & 0.11 & 3 & 6 & 17.56 & 5 & 4 & 0.11 & 4 \\
\hline PSIP1 & 11 & 9.02 & 29 & 14 & 0.02 & 34 & 8 & 12.29 & 19 & 16 & 0.04 & 43 \\
\hline $\mathrm{RCC} 2$ & 18 & 7.76 & 22 & 13 & 0.08 & 16 & 17 & 14.04 & 54 & 16 & 0.03 & 46 \\
\hline SRRT & 12 & 13.11 & 14 & 17 & 0.03 & 19 & 32 & 19.15 & 39 & 30 & 0.02 & 36 \\
\hline TJP2 & 4 & 7.67 & 50 & 5 & 0.13 & 49 & 4 & 16.99 & 25 & 2 & 0.02 & 27 \\
\hline USP39 & 23 & 6.91 & 26 & 19 & 0.03 & 22 & 12 & 17.50 & 13 & 13 & 0.08 & 14 \\
\hline TERF1 & 2 & 4.04 & 2 & 2 & 0.11 & 2 & 6 & 8.02 & 6 & 5 & 0.04 & 5 \\
\hline TERF2 & 6 & 3.46 & 6 & 4 & 0.03 & 4 & 8 & 8.76 & 9 & 6 & 0.05 & 6 \\
\hline HNRNPA1 & 1 & 1.27 & 189 & 1 & 0.93 & 171 & 1 & 5.88 & 181 & 0 & 0.03 & 244 \\
\hline HNRNPM & 41 & 1.53 & 95 & 42 & 0.57 & 107 & 21 & 0.87 & 35 & 48 & 0.68 & 141 \\
\hline
\end{tabular}

these criteria were investigated as putative novel TERRA-associated proteins covering diverse cellular functions. The high-mobility group proteins HMGA1, HMGB1, and HMGB2 had the highest SILAC ratios in both TERRA pull-downs. Because TJP1 has previously been found to be enriched, we thus selected TJP2, which fulfills our requirements. Based on our filter criteria, we further chose a variety of proteins involved in RNA/DNA binding (ZNF691, SRRT, GRSF1), chromatin remodeling (ARID1A), histone acetylation (MORF4L2), DNA-damage signaling/repair (INIP), pre-mRNA splicing (USP39), chromosome condensation (RCC2), metabolism (PC, DBT), and transcriptional coactivation (PSIP1) for the knockdown analysis. Although not among the top-scoring candidates, we included TERF1, TERF2, HNRNPA1, and HNRNPM in our assays because they have well-described functions at telomeres.

\section{Measurements of TERRA level upon candidate knockdown}

The new candidate TERRA interactors that we identified in our screen may be involved in various aspects of TERRA biology, including TERRA expression, stability at telomeres, regulatory roles at telomeres, or signaling functions. First, to test whether some of these candidate proteins are involved in the regulation of TERRA steady-state levels, we used endoribonuclease-prepared shortinterference RNA (esiRNA) (Kittler et al. 2005) for the above selected 19 proteins (Supplemental Fig. 1) and measured the impact on TERRA molecule abundance by quantitative RT-PCR. The cellular TERRA molecules were reverse transcribed with a primer located in the UUAGGG tract. Then, for qPCR experiments, primers were designed in the subtelomeric region, between the telomeric tract and the CpG island promoter of TERRA, generally localized within $2 \mathrm{~kb}$ from the telomere. This, in some instances, allows us to measure the number of TERRA molecules resulting from the transcription of a single telomere. However, since these subtelomeric regions are highly conserved among chromosome ends, it is also possible to design a set of primers that amplifies several chromosome ends averaging the effect of multiple loci. In our qRTPCR experiments, we used a set of primers that, to our knowledge, is specific to $15 q$, and a set of primers that amplifies at least four chromosome ends, namely, 1q-2q-10q-13q. Because DNA sequences are not yet available for all chromosome ends, it is, however, possible that our sets of primers also recognize TERRA molecules from other telomeres.

As expected from previous reports in mice (Schoeftner and Blasco 2008), TERF1 knockdown led to significant down-regulation of TERRA abundance in comparison to a transfection with an esiRNA directed against Renilla luciferase (RLuc) used as negative control in all experiments (Fig. 3A). We can conclude from this result that TERF1 plays a positive role in TERRA transcription and/or stability. It is noteworthy that while 1q-2q-10q-13q TERRA levels are greatly reduced after TERF1 knockdown, the levels of $15 \mathrm{q}$ TERRA remain unaffected. Although we expect TERRA transcription/ stability to be generally regulated the same way at every chromosome end, it is also possible that some factors affect certain TERRA promoters (transcription) or molecules (stability) more than others.

Remarkably, knockdown of half of our candidates led to a decrease in TERRA levels. For $H M G B 2, D B T$, and $P C$, the effect was moderate $(\sim 1.5$-fold increase) but statistically significant $(P<$ 0.05). Knockdown of ARID1A, MORF4L2, PSIP1, RCC2, and USP39 had a stronger effect, reducing TERRA levels by at least twofold for at least one experiment $(P<0.005)$. SRRT, on the other hand, was the only candidate whose knockdown up-regulated TERRA levels both for $1 \mathrm{q}-2 \mathrm{q}-10 \mathrm{q}-13 \mathrm{q}$ as well as $15 \mathrm{q}$ by about threefold $(P=$ 0.027). This effect was comparable to the effect seen upon reduction of HNRNPA1 levels (Fig. 3A), a well-characterized regulator of TERRA (Lopez de Silanes et al. 2010; Flynn et al. 2011). Because TERRA levels are regulated over the cell cycle, with a maximum expression of the RNA in $G_{1}$ and a minimum level in late $S / G_{2}$ (Porro et al. 2010), we wanted to exclude any possible artifact due to cell cycle perturbation. We therefore monitored cell cycle states after knockdown of the candidates and excluded any significant change in cell cycle distribution upon knockdown (Fig. 3B; Supplemental Fig. 2).

Although a thorough study of each candidate is necessary to understand precisely how these proteins regulate TERRA, it is interesting to notice that almost all the proteins affecting TERRA steady-state levels do so by acting positively on TERRA abundance. 
A

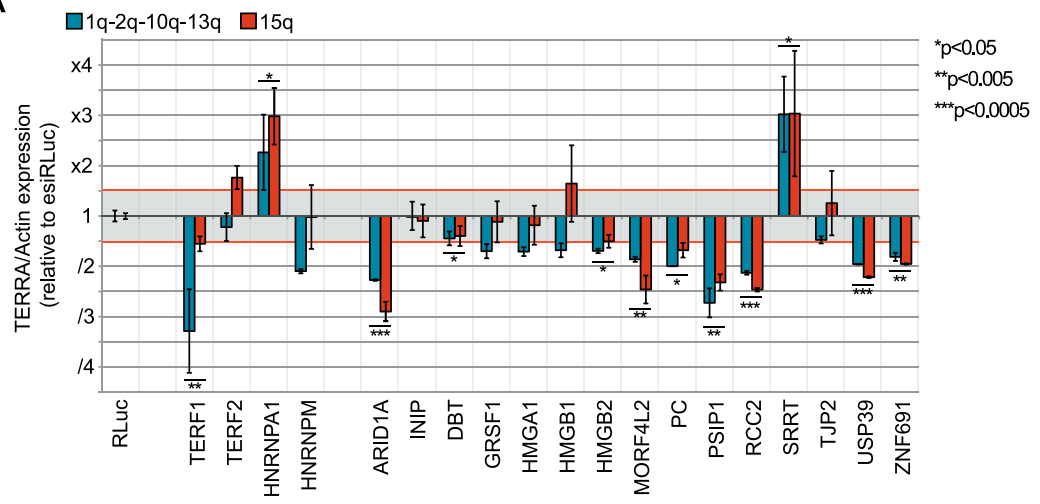

B

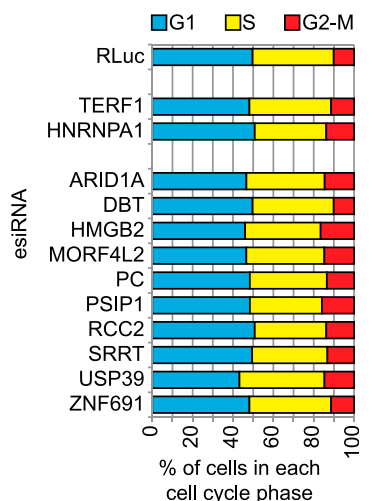

Figure 3. TERRA levels after knockdown of genes encoding candidate proteins. ( $A$ ) Number of TERRA molecules in HeLa Kyoto transfected with the indicated esiRNA, quantified by qRT-PCR at $1 \mathrm{q}-2 \mathrm{q}-10 \mathrm{q}-13 \mathrm{q}$ (blue bars) and $15 \mathrm{q}$ (red bars), normalized to beta actin and compared to levels upon esiRNA transfection against RLuc. Error bars represent standard deviation (SD) between three independent experiments. $\left({ }^{*}\right) P<0.05,\left({ }^{* *}\right) P<0.005,\left({ }^{* * *}\right) P<$ 0.0005 . (B) Percentage of cells in $\mathrm{G}_{1}$ (blue), $\mathrm{S}$ (yellow), and $\mathrm{G}_{2}-\mathrm{M}$ (red) phase, assessed by flow cytometry after BrdU and propidium iodide labeling.

Therefore, transcription and stabilization of TERRA appear to be heavily regulated processes requiring a variety of factors.

\section{Measurement of TERRA abundance at telomeres upon candidate knockdown}

Using cellular fractionation followed by TERRA qRT-PCR, Porro et al. (2010) reported that only $\sim 20 \%$ of total cellular TERRA associate with chromatin, while the remaining $80 \%$ are found in the nucleoplasmatic fraction. SMG proteins have been shown to remove TERRA from chromatin, because knockdown of several SMG proteins increased chromatin-associated TERRA with no effect on global TERRA levels (Azzalin et al. 2007). In this study, Azzalin and colleagues measured chromatin-associated TERRA using RNA-FISH in Triton-extracted cells, a procedure that allows selective detection of chromatin-associated proteins or RNA species, while extracting those that are not associated with chromatin. We relied on the same technique to test whether some of the selected proteins have an impact on TERRA abundance at telomeric chromatin. For better visualization of TERRA, RNA-FISH experiments using locked nucleic acid (LNA) C-rich probes were performed in the 1.3 subclone of HeLa cells that harbors long telomeres of $\sim 15 \mathrm{~kb}$ (Fig. 4A; Takai et al. 2010). To control for telomeric association of TERRA, we combined TERRAFISH with immunofluorescence detection of TERF2, as a marker protein for telomeres (Fig. 4B,C). This confirmed that the vast majority of TERRA foci indeed colocalize with TERF2 (Fig. 4C), and we therefore anticipated that delocalization or relocalization of TERRA from or to telomeres would result in changes in the total intensity of TERRA-FISH foci. As a consequence of either TERRA dilution in the nucleoplasm or nonaccessibility of non-chromatin-associated TERRA molecules, only the RNA molecules that are bound to telomeres are detected in our TERRA-FISH experiments. Hence, TERRA-FISH signals do reflect the amount of telomere-bound TERRA.

In agreement with the general reduction of TERRA levels upon TERF1 depletion, TERRA-FISH foci intensity was equally significantly lower in this setting compared with a control transfection (Fig. 4D). In contrast, we found that TERF2 knockdown increased the intensity of TERRA foci by nearly 1.4-fold (Fig. 4D). Given that by our qPCR measurements we did not detect a significant change in global TERRA levels in TERF2-depleted cells, this suggests that the increased intensity of TERRA foci is indeed due to increased association with telomeres (Fig. 3A). Similarly, in the case of the HNRNPM knockdown, TERRA foci intensity was increased, in agreement with a previous report of relocalization of TERRA to telomeres, while depletion of HNRNPA1 had no major effect (Lopez de Silanes et al. 2010).

Although in most cases knockdown of candidate genes led to a down-regulation of total cellular levels of TERRA, TERRA abundance at telomeres was rarely down-regulated, supporting the view that TERRA transcription and its binding to telomeres are generally regulated independently. For knockdown of ARID1A, HMGB2, PC, PSIP1, RCC2, or USP39, changes in the total cellular levels of TERRA detected by qRT-PCR were not accompanied by significant differences in telomere-associated TERRA (Figs. 3A, 4D), even though several of these candidates, e.g., ARID1A, PSIP1, and RCC2, showed quantitatively similar changes in total TERRA levels compared with TERF1 depletion. Conversely, depletion of HMGA1, HMGB1, both from the high-mobility group family, or ZNF691, a zinc finger protein with unknown function, increased TERRA abundance at telomeres without affecting global TERRA levels. Hence, the three latter proteins may be involved, directly or indirectly, in TERRA removal from telomeres.

In contrast, depletion of SRRT, a mediator between the nuclear RNA cap-binding complex and the miRNA processing machinery (Gruber et al. 2009), was found to increase both global TERRA levels and TERRA abundance at telomeres (Figs. 3A, 4D). Knockdown of MORF4L2, a component of the NuA4 histone acetyltransferase complex (Cai et al. 2003), was associated with a coinciding reduction of TERRA at the global cellular level and at telomeres (Figs. 3A, 4D). This suggests that SRRT and MORF4L2 represent factors specifically impacting on the stability of TERRA molecules associated with telomeres. Finally, ZNF691 represents an intriguing outlier from these two patterns. Juxtaposed to the reduction of general TERRA levels observed upon ZNF691 esiRNA knockdown (Fig. 3A), TERRA association with telomeres was increased by $\sim 40 \%$ upon ZNF691 depletion (Fig. 4D).

In sum, 12 out of 15 proteins with a high SILAC ratio in our mass spectrometric screen resulted in phenotypic changes on global TERRA abundance and/or TERRA association with telomeric chromatin upon knockdown, highlighting that our screening approach has identified new candidate proteins involved in TERRA metabolism. 
A
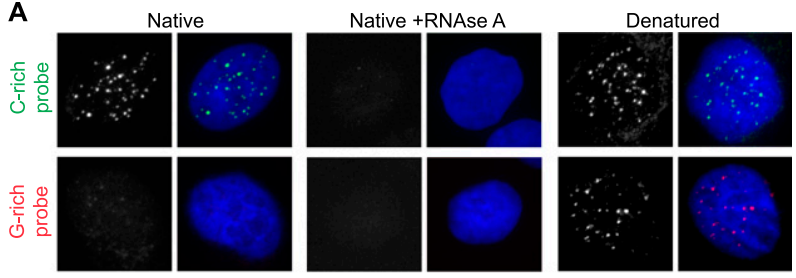

B

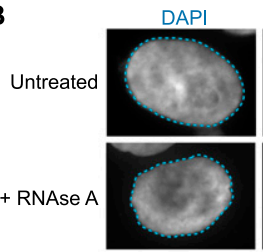

TERF2
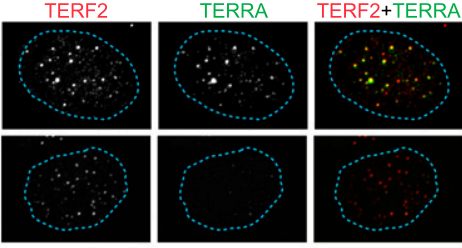

C
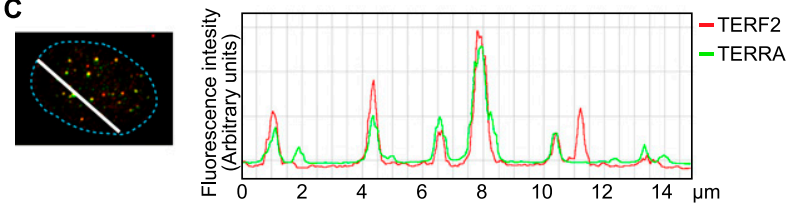

D

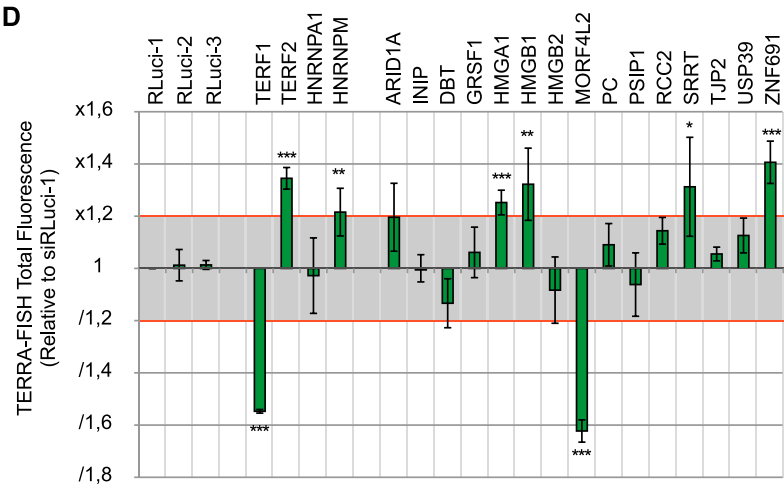

Figure 4. Quantitative TERRA-FISH after knockdown of the candidates in HeLa 1.3. (A) RNA-FISH with CCCTAA (green, upper panel) or TTAGGC (red, lower panel) LNA probes, including RNase $\mathrm{A}$ and denatured controls. (B) TERRA-FISH (green) coupled with TERF2 immunofluorescence (red). (C) Representative example of 10 independent experiments of signal intensity correlation between TERF2 (red) and TERRA (green) following the indicated white line. $(D)$ Quantification of TERRA foci in HeLa 1.3 after knockdown of the candidate genes, relative to RLuc-1. Error bars indicate SD between three independent experiments. $\left(^{*}\right) P<0.05,\left({ }^{* *}\right) P<0.01$, $\left({ }^{* * *}\right) P<0.001$.

\section{Discussion}

Employing quantitative high-resolution mass spectrometry, we found significant enrichment of 115 proteins with TERRA compared with our control RNA. In addition to new candidate TERRA interactors, we recovered most candidate binders from two previous studies. Of the enriched TERRA-associated proteins, a large fraction forms a STRING network among itself, whose structure suggests that TERRA is involved in different functional processes and/or regulated by those (Fig. 2). To characterize a putative function of our candidates in TERRA biology, we performed an esiRNA-based knockdown analysis on a subset of the enriched proteins. This RNAi analysis revealed changes in TERRA levels and/ or telomeric localization for nearly all selected proteins.

Depletion of TERF1 and MORF4L2 led to a global decrease of cellular TERRA levels that proportionally decreased the amount of telomere-associated TERRA, suggesting that these two proteins may be required for both TERRA transcription (or stability) and telomere binding. Alternatively, both proteins could selectively impact the stability of telomere-bound TERRA molecules. Interestingly, MORF4L2 is a component of the NuA4 histone acetyltransferase complex previously shown to be important in establishing telomeric heterochromatin boundaries in budding yeast (Babiarz et al. 2006). In view of recent data reporting a negative impact of telomeric heterochromatin on TERRA expression (Arnoult et al. 2012), we anticipate that loss of MORF4L2 may increase telomeric repressive histone marks and/or lead to the spreading of telomeric repressive marks onto TERRA promoters, thus breaking down the natural barriers of human telomeric heterochromatin and presents an interesting hypothesis to be tested in the future. ARID1A is another of our candidates whose knockdown led to decreased overall levels of TERRA. It has been established as a non-sequence-specific DNA binding protein that is part of the BAF-type SWI-SNF nucleosome remodeling complex (Dallas et al. 1998). While ARID1A is able to recruit the SWI/SNF complex to heterochromatin, it allows the transcriptional activation of normally silenced chromatin. Therefore, it is tempting to speculate that recruitment of ARID1A by TERRA could induce the remodeling of telomeric/subtelomeric chromatin and could activate TERRA transcription.

On the other hand, SRRT depletion increased both global and telomere-associated TERRA levels. SRRT has been recently implicated in pri-miRNA processing in humans by interaction with the nuclear cap-binding complex (CBC) (Gruber et al. 2009). In plants, SRRT was reported to be involved in cell defense against RNA viruses through its ability to modulate the siRNA pathway (Sabin et al. 2009). Hence, SRRT may destabilize TERRA through its ability to modulate microprocessor activity and/or to induce its translocation to the cytoplasm, where TERRA is degraded. In our quantitative MS screen, we found members of the high-mobility group protein family among the most highly enriched candidates. These proteins have already been linked to DNA-damage recognition (Prasad et al. 2007), but have also been investigated with respect to telomere biology. While $H M G B 1^{-1-}$ MEFs display shorter telomeres and reduced telomerase activity, the effect is reversed for HMGB2 (Polanska et al. 2012). In our experiments, we also observe this functional difference between HMGB1 and HMGB2 for TERRA regulation. While HMGA1 and HMGB1 depletion led to increased association of TERRA with telomeres without affecting overall cellular TERRA levels, depletion of HMGB2 was followed by a mild depletion of TERRA levels without affecting the amount of telomere-bound TERRA.

Three of the candidate proteins analyzed-INIP, GRSF1, and TJP2-had no effect on overall TERRA levels and TERRA localization at the telomere, and their biological importance in TERRA biology remains open at the moment. Because our follow-up does not cover all aspects of TERRA regulation, they may still have important functions to be discovered in the future. Interestingly, INIP forms heterotrimeric complexes with INTS3 and the ssDNA binding proteins NABP1 (also known as SSB2) or NABP2 (also known as SSB1), which are very recently reported to be important in the protection of newly replicated telomeres (Gu et al. 2013). We envision that TERRA could play a role in the recruitment of both complexes at telomeres, possibly through its interaction with INIP.

Our quantitative interaction study revealed several TERRAassociated proteins not detected in previous experiments. We performed a first general characterization of 15 of them and observed changes in TERRA localization and/or expression levels for 
the large majority of the candidates. Notably, effect sizes for these proteins were within the range of previously characterized important TERRA regulators and known telomere binding proteins such as TERF1 and TERF2. Our screen implies that multiple major cellular processes such as chromatin modification, RNA degradation, transcription, and DNA replication are connected to TERRA regulation. Thus, our results indicate that the characterization of important regulators of TERRA is by no means completed and that the TERRA-associated proteins reported here should be a valuable resource for the field, enabling a more systematic analysis of its regulation in the future.

\section{Methods}

\section{SILAC labeling and nuclear extract preparation}

HeLa S3 cells were SILAC-labeled in RPMI 1640 (-Arg, -Lys) medium containing 10\% dialyzed fetal bovine serum (PAA), 84 $\mathrm{mg} / \mathrm{L}{ }^{13} \mathrm{C}_{6}{ }^{15} \mathrm{~N}_{4}$ L-arginine (Euriso-Top), and $40 \mathrm{mg} / \mathrm{L}{ }^{13} \mathrm{C}_{6}{ }^{15} \mathrm{~N}_{2}$ L-lysine (Euriso-Top) or the corresponding concentration of unlabeled amino acids (Sigma-Aldrich), respectively, for at least 10 cell divisions. Nuclear extracts were prepared essentially as previously described. In short, cells were harvested, resuspended in low osmolaric buffer $(10 \mathrm{mM} \mathrm{KCl}, 10 \mathrm{mM}$ HEPES/KOH at $\mathrm{pH} 7.9$, $1.5 \mathrm{mM} \mathrm{MgCl}_{2}, 0.1 \%$ IGEPAL CA630, $0.5 \mathrm{mM}$ DTT, protein inhibitor complete [Roche]), and allowed to swell for $15 \mathrm{~min}$ prior to 30 strokes with pestle B in a douncer. The nuclei were pelleted by centrifugation at $3000 \mathrm{~g}$ for $15 \mathrm{~min}$, washed once with PBS, and resuspended in high osmolaric buffer $(420 \mathrm{mM} \mathrm{NaCl}, \mathrm{HEPES} / \mathrm{KOH}$ at pH 7.9, $2 \mathrm{mM} \mathrm{MgCl}_{2}, 0.2 \mathrm{mM}$ EDTA at pH 8.0, 0.1\% IGEPAL CA630, $0.5 \mathrm{mM}$ DTT, protein inhibitor complete [Roche]). Lysis proceeded under slight agitation for $1 \mathrm{~h}$ at $4^{\circ} \mathrm{C}$. Cell debris was separated by $15 \mathrm{~min}$ of centrifugation at $13,000 \mathrm{~g}$ in a tabletop centrifuge. Nuclear extracts were shock frozen in liquid nitrogen and stored at $-80^{\circ} \mathrm{C}$ until use.

\section{RNA pull-downs with SILAC nuclear extracts}

Twenty-five micrograms of biotinylated TERRA RNA (UUAGGG) 8 or biotinylated control RNA (GUGUGA) 8 was bound to $50 \mu \mathrm{L}$ of magnetic streptavidin Dynabeads C1 (Invitrogen) in RNA binding buffer containing $50 \mathrm{mM}$ HEPES (pH 7.6), 0.5\% IGEPAL CA 630, $10 \mathrm{mM} \mathrm{MgCl}_{2}, 4 \mathrm{M}$ urea, and $250 \mathrm{mM} \mathrm{NaCl}$ for $1 \mathrm{~h}$ at $4^{\circ} \mathrm{C}$ on a rotation wheel. Beads were washed three times with RNA wash buffer (50 mM HEPES at pH 7.6, 0.5\% IGEPAL CA630, $10 \mathrm{mM}$ $\mathrm{MgCl}_{2}, 250 \mathrm{mM} \mathrm{NaCl}_{2}$ ) before incubation with $400 \mu \mathrm{g}$ of nuclear extract, 40 units of RNase Inhibitor (Fermentas), and $20 \mu \mathrm{g}$ of yeast tRNA for $1 \mathrm{~h}$ at $4^{\circ} \mathrm{C}$ on a rotation wheel. After washing with $50 \mathrm{mM}$ HEPES/HCl (pH 7.6), 0.5\% IGEPAL CA630, $10 \mathrm{mM} \mathrm{MgCl}_{2}, 250 \mathrm{mM}$ (or $1 \mathrm{M}$ ) $\mathrm{NaCl}$ fractions were combined and eluted in SDS loading dye for $15 \mathrm{~min}$ at $70^{\circ} \mathrm{C}$ under vigorous shaking.

\section{Mass spectrometry and data analysis}

Pull-downs were separated on a $4 \%-12 \%$ NOVEX gradient SDS gel (Life Technologies) for $1 \mathrm{~h}$ at $170 \mathrm{~V}$ in $1 \times$ MOPS buffer (Life Technologies). Proteins were fixated and stained with the Colloidal Blue Staining Kit (Life Technologies). The gel lane was cut into eight slices, minced, and destained with a $50 \%$ ethanol $/ 50 \mathrm{mM}$ ammonium bicarbonate solution. Proteins were reduced in $10 \mathrm{mM}$ DTT for $1 \mathrm{~h}$ at $56^{\circ} \mathrm{C}$ and then alkylated with $55 \mathrm{mM}$ iodoacetamide for $1 \mathrm{~h}$ at room temperature. Proteins were digested with trypsin (Promega) overnight at $37^{\circ} \mathrm{C}$. Peptides were extracted from the gel using acetonitrile, which was subsequently removed in a concentrator (Eppendorf) and loaded on stage tips for storage. For mass spectrometric analysis, peptides were separated on a C18 column with $75-\mu \mathrm{m}$ diameter packed with $3-\mu \mathrm{m}$ Reprosil beads (Dr. Maisch) mounted to a EASY HPLC (Thermo Fisher) and sprayed online into an Orbitrap-XL mass spectrometer (Thermo Fisher). We used a 120 -min gradient from $2 \%$ to $60 \%$ acetonitrile in $0.5 \%$ acetic acid at a flow of $200 \mathrm{~nL} / \mathrm{min}$. The mass spectrometer was operated with CID fragmentation for the top 10 data-dependent MS/MS per full scan. Mass spectrometry raw data were searched using the Andromeda search engine (Cox et al. 2011) integrated into MaxQuant suite 1.2.2.0 (Cox and Mann 2008) using the IPI human database v3.68. Carbamidomethylation at cysteine was set as fixed modification while methionine oxidation and protein $\mathrm{N}$-acetylation were considered as variable modifications. The search was performed with an initial mass tolerance of $7 \mathrm{ppm}$ mass accuracy for the precursor ion and 0.5 Da (CID) for the MS/MS spectra. Search results were processed with MaxQuant and identifications up to a false discovery rate of 0.01 were accepted. For the STRING analysis (version 9.05), we used the complete set of functional STRING associations including genomic context, high-throughput experiments, coexpression, and previous knowledge (text mining).

\section{Production of endonuclease-prepared short-interference RNA (esiRNA)}

esiRNAs were produced by Eupheria Biotech applying previously published protocols (Kittler et al. 2005). Briefly, optimal regions for designing esiRNAs were chosen using the Deqor design algorithm (Henschel et al. 2004) in order to fulfill two criteria: to obtain the most efficient silencing trigger in terms of silencing efficiency, and to get the lowest chances to cross-silence other genes. The most favorable fragments were used to design gene-specific primers using the Primer3 algorithm (http://frodo.wi.mit.edu/ cgi-bin/primer3/primer3_www.cgi), and the resulting PCR products were sequence verified on an Applied Biosystems 3730 Genetic Analyzer (Applied Biosystems) according to the manufacturer's instructions. EsiRNA cDNA target sequences can be found in Supplemental Table 2.

\section{Cell culture and transfection for esiRNA screen}

HeLa 1.2.11 clone 1.3 (deLange Laboratory, Rockefeller University) were grown at $37^{\circ} \mathrm{C}$ with $5 \% \mathrm{CO}_{2}$ in DMEM media (GIBCO, Invitrogen) supplemented with $10 \%$ fetal bovine serum (HiClone, Perbio Science), 1\% nonessential amino acids (Invitrogen), and penicillin/streptomycin. Cells were plated in six-well plates (45,000 HeLa cells per well), and transfected $24 \mathrm{~h}$ later with $2 \mu \mathrm{g}$ of esiRNA and $30 \mu \mathrm{L}$ of oligofectamine (Invitrogen) in Opti-MEM (Invitrogen). Five hours after transfection, cells were washed and media was replaced; cells were finally collected for RNA extraction $48 \mathrm{~h}$ later.

\section{Quantitative real-time PCR}

For quantification of mRNA transcript levels upon individual esiRNA knockdown, RNA was extracted with the RNeasy kit (QIAGEN), including DNase I digestion. cDNA was synthesized from the eluted RNA using the SuperScript III and an oligo(dT) primer (Life Technologies) according to the manufacturer's instructions. qPCR primers (Metabion) (Supplemental Table 2) were used at a $70 \mathrm{nM}$ concentration together with the Absolute qPCR SYBR Green mix (Abgene) on a CFX96 Real-Time System (Bio-Rad). Target gene mRNA levels were normalized against quantification of GAPDH mRNA levels for housekeeping. 


\section{TERRA qRT-PCR measurements}

TERRA quantitative real-time PCR was performed as described previously with slight modifications (Porro et al. 2010). Briefly, RNA was extracted using the Nucleospin RNA II Kit (Macherey Nagel), with in-column DNA digestion. Two micrograms of RNA was reverse transcribed with beta actin- and telomere-specific primers (Supplemental Table 2) at $55^{\circ} \mathrm{C}$ using SuperScript III Reverse Transcriptase (Invitrogen). For SYBR Green reactions, 1q-2q10q-13q and 15q specific transcripts were analyzed using specific primers (Supplemental Table 2) and normalized against beta actin levels. Real-time PCR was performed using the Kapa SYBR Fast qPCR Kit master mix (Kapa Biosystems). A -RT control was performed each time to control for efficient DNA digestion.

\section{TERRA-fluorescence in situ hybridization (FISH)}

TERRA-FISH was performed as described previously with some modifications (Azzalin et al. 2007). HeLa 1.3 cells were transfected with esiRNA and seeded on four-well cell culture slides (VWR). Forty-eight hours later, cells were permeabilized for $7 \mathrm{~min}$ on ice with CSK permeabilization buffer (10 mM Pipes at pH 7.0, $100 \mathrm{mM}$ $\mathrm{NaCl}, 300 \mathrm{mM}$ sucrose, $3 \mathrm{mM} \mathrm{MgCl}$, $0.5 \%$ Triton X-100, $10 \mathrm{mM}$ Ribonucleoside Vanadyl Complex), fixed for $10 \mathrm{~min}$ at room temperature with $3.7 \%$ formaldehyde/PBS, and rinsed three times in PBS. Cells were then dehydrated with a successive $70 \%-85 \%-$ $100 \%$ ethanol bath and hybridized for $1 \mathrm{~h}$ at $37^{\circ} \mathrm{C}$ with hybridization mix containing $400 \mathrm{nM}$ of the LNA G-rich probe (TAM)GGGTtAGGGttAGgGTTAGGGttAGGGttAGGG(TAM), where small letters represent LNA bases, or C-rich probe (FAM)CCC

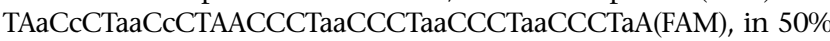
deionized formamide, $10 \%$ dextran sulfate (Millipore), $2 \times$ SSC, $2 \mathrm{mg} / \mathrm{mL}$ BSA (NEB), and $10 \mathrm{mM} \mathrm{RVC} \mathrm{(NEB).} \mathrm{Cells} \mathrm{were} \mathrm{then} \mathrm{rinsed}$ three times for $5 \mathrm{~min}$ in $0.1 \times \mathrm{SSC}$ at $60^{\circ} \mathrm{C}$ and once for $5 \mathrm{~min}$ in $2 \times$ SSC at room temperature. Slides were then dehydrated and mounted in mounting media containing DAPI. For RNase control treatment, cells were treated as before with the following exceptions: Permeabilization was performed in CSK buffer without $\mathrm{RVC}$, and after fixation, cells were incubated for $1 \mathrm{~h}$ at $37^{\circ} \mathrm{C}$ with $1 \mathrm{mg} / \mathrm{mL}$ RNase A in PBS. A denaturation control was performed with $4 \mathrm{~min}$ at $78^{\circ} \mathrm{C}$ following cell fixation.

\section{Microscopy and quantitative analysis}

Images of nuclei were captured with a confocal Cell Observer Spinning Disc microscope (Zeiss) using AxioVision software. TERRA, TERF2, and telomeric foci were quantified using ImageJ software (National Institutes of Health). Each focus was measured using local maxima determination with a noise tolerance of 40 , on z-stack projected images. Thirty nuclei from at least seven different images were quantified for each condition, and the background of images was subtracted to each focus. Histograms represent the average of three independent experiments, and error bars represent standard deviation between experiments.

\section{FACS analysis}

FACS was essentially done as previously described (Arnoult et al. 2009). In short, $48 \mathrm{~h}$ after transfection, cells were trypsinized, resuspended in $500 \mu \mathrm{L}$ of PBS, and fixed with $1.5 \mathrm{~mL}$ of ethanol. Fixed cells were then incubated first in $0.05 \%$ pepsin A (SigmaAldrich) and $30 \mathrm{mM} \mathrm{HCl}$ for $20 \mathrm{~min}$ at $37^{\circ} \mathrm{C}$ and then in $2 \mathrm{M} \mathrm{HCl}$ for $20 \mathrm{~min}$ at room temperature. Cells were washed $1 \times$ in PBS, $1 \times$ in BU buffer $(0.5 \%$ normal goat serum, $0.5 \%$ Tween 20 , and $20 \mathrm{mM}$ HEPES in PBS) and incubated for $45 \mathrm{~min}$ at room temperature with rat anti-BrdU antibody (clone BU1/75, AbD Serotec) diluted at 1:50 in BU buffer. After washing with PBS, cells were incubated for $30 \mathrm{~min}$ at room temperature with secondary antibody (Goat antirat-FITC conjugate 3030-02, Southern Biotech) diluted at 1:50 in BU buffer. After another wash with PBS, cells were pelleted and resuspended into PBS containing $30 \mu \mathrm{g} / \mathrm{mL}$ propidium iodide (Sigma-Aldrich) and $200 \mu \mathrm{g} / \mathrm{mL}$ RNase A (Sigma-Aldrich). Flow cytometry was performed using a Becton Dickinson FACSort flow cytometer, and data were analyzed with FlowJo software.

\section{Data access}

The mass spectrometry proteomics data have been submitted to the ProteomeXchange Consortium (http://proteomecentral. proteomexchange.org) via the PRIDE partner repository (Vizcaíno et al. 2013) with the data set identifier PXD000342.

\section{Acknowledgments}

We thank Igor Paron and Korbinian Mayr (Department of Proteomics and Signal Transduction, MPI for Biochemistry) for technical assistance, Sebastian Rose and Romy Heinze (Eupheria Biotech) for providing esiRNAs, and Bernd Haas (core facility, MPI for Biochemistry) for synthesis of RNA oligonucleotides. The HeLa 1.3 cell line was a gift from Titia deLange (Rockefeller University). We thank the PRIDE team for their support with data storage. Work in the Mann laboratory was supported by grants from the Max Planck Society and the European Union (HEALTH-F4-2008-201648). The Buchholz laboratory was supported by the TU Dresden, and grants from the Deutsche Forschungsgemeinschaft (SFB 655, BU 1400/5-1) and the Saxon Staatsministerium für Wissenschaft und Kunst (SMWK).

\section{References}

Arnoult N, Saintome C, Ourliac-Garnier I, Riou JF, Londono-Vallejo A. 2009 Human POT1 is required for efficient telomere C-rich strand replication in the absence of WRN. Genes Dev 23: 2915-2924.

Arnoult N, Van Beneden A, Decottignies A. 2012. Telomere length regulates TERRA levels through increased trimethylation of telomeric H3K9 and HP1 $\alpha$. Nat Struct Mol Biol 19: 948-956.

Arora R, Brun CM, Azzalin CM. 2012. Transcription regulates telomere dynamics in human cancer cells. RNA 18: 684-693.

Azzalin CM, Reichenbach P, Khoriauli L, Giulotto E, Lingner J. 2007. Telomeric repeat containing RNA and RNA surveillance factors at mammalian chromosome ends. Science 318: 798-801.

Babiarz JE, Halley JE, Rine J. 2006. Telomeric heterochromatin boundaries require NuA4-dependent acetylation of histone variant H2A.Z in Saccharomyces cerevisiae. Genes Dev 20: 700-710.

Baltz AG, Munschauer M, Schwanhausser B, Vasile A, Murakawa Y, Schueler M, Youngs N, Penfold-Brown D, Drew K, Milek M, et al. 2012. The mRNA-bound proteome and its global occupancy profile on proteincoding transcripts. Mol Cell 46: 674-690.

Butter F, Scheibe M, Morl M, Mann M. 2009. Unbiased RNA-protein interaction screen by quantitative proteomics. Proc Natl Acad Sci 106: $10626-10631$.

Cai Y, Jin J, Tomomori-Sato C, Sato S, Sorokina I, Parmely TJ, Conaway RC, Conaway JW. 2003. Identification of new subunits of the multiprotein mammalian TRRAP/TIP60-containing histone acetyltransferase complex. J Biol Chem 278: 42733-42736.

Cox J, Mann M. 2008. MaxQuant enables high peptide identification rates, individualized p.p.b.-range mass accuracies and proteome-wide protein quantification. Nat Biotechnol 26: 1367-1372.

Cox J, Neuhauser N, Michalski A, Scheltema RA, Olsen JV, Mann M. 2011. Andromeda: A peptide search engine integrated into the MaxQuant environment. J Proteome Res 10: 1794-1805.

Dallas PB, Cheney IW, Liao DW, Bowrin V, Byam W, Pacchione S, Kobayashi R, Yaciuk P, Moran E. 1998. p300/CREB binding protein-related protein p270 is a component of mammalian SWI/SNF complexes. Mol Cell Biol 18: $3596-3603$.

Deng Z, Norseen J, Wiedmer A, Riethman H, Lieberman PM. 2009. TERRA RNA binding to TRF2 facilitates heterochromatin formation and ORC recruitment at telomeres. Mol Cell 35: 403-413. 
Farnung BO, Brun CM, Arora R, Lorenzi LE, Azzalin CM. 2012. Telomerase efficiently elongates highly transcribing telomeres in human cancer cells. PLOS ONE 7: e35714.

Flynn RL, Centore RC, O'Sullivan RJ, Rai R, Tse A, Songyang Z, Chang S, Karlseder J, Zou L. 2011. TERRA and hnRNPA1 orchestrate an RPA-toPOT1 switch on telomeric single-stranded DNA. Nature 471: 532-536.

Gruber JJ, Zatechka DS, Sabin LR, Yong J, Lum JJ, Kong M, Zong WX, Zhang Z, Lau CK, Rawlings J, et al. 2009. Ars2 links the nuclear cap-binding complex to RNA interference and cell proliferation. Cell 138: 328-339.

$\mathrm{Gu}$ P, Deng W, Lei M, Chang S. 2013. Single strand DNA binding proteins 1 and 2 protect newly replicated telomeres. Cell Res 23: 705-719.

Henschel A, Buchholz F, Habermann B. 2004. DEQOR: A web-based tool for the design and quality control of siRNAs. Nucleic Acids Res 32: W113W120.

Kittler R, Heninger AK, Franke K, Habermann B, Buchholz F. 2005. Production of endoribonuclease-prepared short interfering RNAs for gene silencing in mammalian cells. Nat Methods 2: 779-784.

Klass DM, Scheibe M, Butter F, Hogan GJ, Mann M, Brown PO. 2013. Quantitative proteomic analysis reveals concurrent RNA-protein interactions and identifies new RNA-binding proteins in Saccharomyces cerevisiae. Genome Res 23: 1028-1038.

Lopez de Silanes I, Stagno d'Alcontres M, Blasco MA. 2010. TERRA transcripts are bound by a complex array of RNA-binding proteins. Nat Commun 1: 33.

Mann M. 2006. Functional and quantitative proteomics using SILAC. Natl Rev 7: 952-958.

Nergadze SG, Farnung BO, Wischnewski H, Khoriauli L, Vitelli V, Chawla R, Giulotto E, Azzalin CM. 2009. CpG-island promoters drive transcription of human telomeres. RNA 15: 2186-2194.

Ong SE. 2010. Unbiased identification of protein-bait interactions using biochemical enrichment and quantitative proteomics. Cold Spring Harb Protoc 2010: pdb.prot5400.

Palm W, de Lange T. 2008. How shelterin protects mammalian telomeres Annu Rev Genet 42: 301-334.

Pfeiffer V, Lingner J. 2012. TERRA promotes telomere shortening through exonuclease 1-mediated resection of chromosome ends. PLoS Genet 8: 1002747.

Polanska E, Dobsakova Z, Dvorackova M, Fajkus J, Stros M. 2012. HMGB1 gene knockout in mouse embryonic fibroblasts results in reduced telomerase activity and telomere dysfunction. Chromosoma 121: 419-431.
Porro A, Feuerhahn S, Reichenbach P, Lingner J. 2010. Molecular dissection of telomeric repeat-containing RNA biogenesis unveils the presence of distinct and multiple regulatory pathways. Mol Cell Biol 30: 48084817.

Prasad R, Liu Y, Deterding LJ, Poltoratsky VP, Kedar PS, Horton JK, Kanno S, Asagoshi K, Hou EW, Khodyreva SN, et al. 2007. HMGB1 is a cofactor in mammalian base excision repair. Mol Cell 27: 829-841.

Redon S, Reichenbach P, Lingner J. 2010. The non-coding RNA TERRA is a natural ligand and direct inhibitor of human telomerase. Nucleic Acids Res 38: 5797-5806.

Sabin LR, Zhou R, Gruber JJ, Lukinova N, Bambina S, Berman A, Lau CK, Thompson CB, Cherry S. 2009. Ars2 regulates both miRNA- and siRNAdependent silencing and suppresses RNA virus infection in Drosophila. Cell 138: 340-351.

Scheibe M, Butter F, Hafner M, Tuschl T, Mann M. 2012. Quantitative mass spectrometry and PAR-CLIP to identify RNA-protein interactions. Nucleic Acids Res. doi: 10.1093/nar/gks746.

Schoeftner S, Blasco MA. 2008. Developmentally regulated transcription of mammalian telomeres by DNA-dependent RNA polymerase II. Nat Cell Biol 10: 228-236.

Szklarczyk D, Franceschini A, Kuhn M, Simonovic M, Roth A, Minguez P, Doerks T, Stark M, Muller J, Bork P, et al. 2011. The STRING database in 2011: Functional interaction networks of proteins, globally integrated and scored. Nucleic Acids Res 39: D561-D568.

Takai KK, Hooper S, Blackwood S, Gandhi R, de Lange T. 2010. In vivo stoichiometry of shelterin components. J Biol Chem 285: 1457-1467.

Tsai BP, Wang X, Huang L, Waterman ML. 2011. Quantitative profiling of in vivo-assembled RNA-protein complexes using a novel integrated proteomic approach. Mol Cell Proteomics 10: M110.007385.

Vizcaíno JA, Côté RG, Csordas A, Dianes JA, Fabregat A, Foster JM, Griss J, Alpi E, Birim M, Contell J, et al. 2013. The PRoteomics IDEntifications (PRIDE) database and associated tools: Status in 2013. Nucleic Acids Res 41: D1063-D1069.

Ward AM, Bidet K, Yinglin A, Ler SG, Hogue K, Blackstock W, Gunaratne J, Garcia-Blanco MA. 2011. Quantitative mass spectrometry of DENV-2 RNA-interacting proteins reveals that the DEAD-box RNA helicase DDX6 binds the DB1 and DB2 3' UTR structures. RNA Biol 8: 1173-1186.

Received November 13, 2012; accepted in revised form July 30, 2013. 


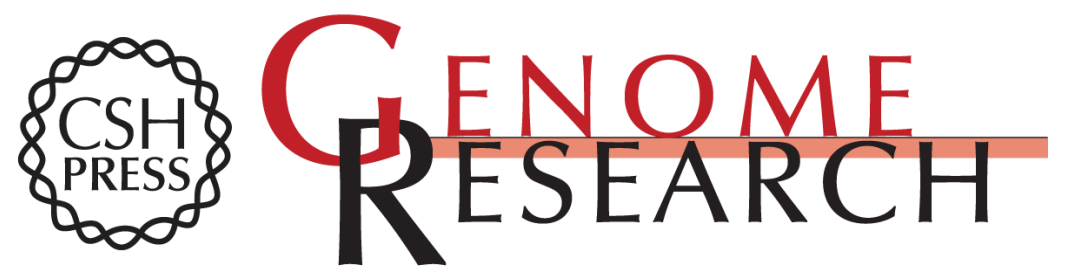

\section{Quantitative interaction screen of telomeric repeat-containing RNA reveals novel TERRA regulators}

Marion Scheibe, Nausica Arnoult, Dennis Kappei, et al.

Genome Res. 2013 23: 2149-2157 originally published online August 6, 2013

Access the most recent version at doi:10.1101/gr.151878.112

Supplemental Material

References

Creative

Commons

License

Email Alerting

Service
http://genome.cshlp.org/content/suppl/2013/09/24/gr.151878.112.DC1

This article cites 36 articles, 13 of which can be accessed free at: http://genome.cshlp.org/content/23/12/2149.full.html\#ref-list-1

This article is distributed exclusively by Cold Spring Harbor Laboratory Press for the first six months after the full-issue publication date (see

http://genome.cshlp.org/site/misc/terms.xhtml). After six months, it is available under a Creative Commons License (Attribution-NonCommercial 3.0 Unported), as described at http://creativecommons.org/licenses/by-nc/3.0/.

Receive free email alerts when new articles cite this article - sign up in the box at the top right corner of the article or click here.

\section{Affordable, Accurate Sequencing.}

To subscribe to Genome Research go to:

https://genome.cshlp.org/subscriptions 